\title{
Erratum to: Higher Education in the High North
}

Marit Sundet, Per-Anders Forstorp and Anders Örtenblad

\section{Erratum to:}

M. Sundet et al. (eds.),

Higher Education in the High North,

Higher Education Dynamics 48,

https://doi.org/10.1007/978-3-319-56832-4

The original version of this book was published without the Acknowledgements section. It is added in the current version. 